\title{
O EFEITO-FRONTEIRA: UMA ANÁLISE DO COMÉRCIO CATARINENSE
}

\author{
Vanessa Grüdtner' \\ Beatriz Scheerman dos Santos Gonçalves²
}

\begin{abstract}
Resumo
O presente artigo analisa o efeito-fronteira do Estado de Santa Catarina para o ano de 2010. A estimação foi realizada através do modelo gravitacional para Santa Catarina, com os demais 25 estados brasileiros, o Distrito Federal e 120 países. É possível concluir que o comércio interestadual é mais significativo para o Estado de Santa Catarina do que o comércio internacional. Os resultados econométricos indicam que Santa Catarina transaciona aproximadamente 32 vezes mais com os demais entes federativos brasileiros do que com outros países.
\end{abstract}

Palavras-chave: efeito-fronteira, modelo gravitacional, comércio internacional, comércio intranacional, Santa Catarina.

Classificação JEL: C2; F1.

\section{INTRODUÇÃO}

O comércio internacional tem cada vez maior importância no cenário mundial globalizado e o Brasil vem, desde a abertura comercial, procurando se inserir nessa dinâmica. Com a repercussão destes fatos, tanto no Brasil como no mundo, emergiram estudos, a partir da década de 90, com vistas a explicar e mensurar em que proporção o comércio interno era mais for-

Graduada em Ciências Econômicas pela Universidade do Estado de Santa Catarina. E-mail: vgrudtner@gmail.com 2 Graduada em Ciências Econômicas pela Universidade do Estado de Santa Catarina. E-mail: beascheerman@gmail. com 
talecido e vantajoso para um país do que seu fluxo com outros países. Este fenômeno foi, no contexto, denominado efeito-fronteira.

Ao longo dos anos surgiram distintas tentativas de formulação de modelos que pudessem explicar a dinâmica do comércio internacional através do efeito-fronteira. O primeiro trabalho foi realizado por estudiosos canadenses, MacCallum, 1995 e Helliwell, 1998, onde se incluiu, partindo do modelo gravitacional de Newton adaptado para as teorias do comércio, incluindo, com o intuito de observar as discrepâncias entre o comércio no âmbito interno dos países (entre estados ou províncias) e o comércio realizado com outros países, uma variável binária.

O que se pretende, com este estudo, é analisar o efeito-fronteira do estado de Santa Catarina com os 25 estados e distrito federal do Brasil e com outros países. Para tanto, foram utilizados dados dos fluxos comerciais de 2010 e foi elaborado um modelo de estimação do efeito baseado no PIB das localidades, na distância entre elas e no fato de serem Estados brasileiros ou países.

Este artigo é dividido em quatro seções além desta introdução. A seção 2 apresenta uma revisão teórica sobre o modelo. A seção 3 descreve a metodologia utilizada para a estimação dos parâmetros do modelo. Em seguida, a seção 4 delineia os resultados alcançados e a seção 5 conclui o estudo.

\section{O MODELO GRAVITACIONAL E O EFEITO-FRONTEIRA: UMA REVISÃO}

\section{O Modelo Gravitacional}

A Lei da Gravidade Universal, postulada por Newton, afirma que entre dois corpos existe uma força de atração que é uma relação diretamente proporcional entre o produto da massa dos corpos e inversamente proporcional ao quadrado da distância entre eles. Tal relação pode ser expressa pela seguinte equação, na qual FG é a força de gravitacional, $m_{1}$ e $m_{2}$ são as massas dos corpos, $\mathrm{G}$ é a Constante Gravitacional e d é a distância:

$$
F G=\frac{G\left(m_{1} \cdot m_{2}\right)}{d^{2}}
$$


É, porém, interessante notar que o desenvolvimento desta teoria revolucionou não somente os conhecimentos da física, mas tem sido utilizada em diversas outras áreas do conhecimento. Interessa-nos a sua adaptação e aplicação às ciências sociais e ainda sua incorporação, desde a década de 1960, às visões econômicas concernentes ao comércio internacional. Os primeiros autores a abordar este tema voltado para o comercio foram Tinbergen (1962) e Poyhonen (1963), eles começaram a desenvolver esta aplicabilidade a fim de definir a influência da distância e da renda nos fluxos comerciais entre países. O diferencial do modelo de Newton está na força de atração que é exercida com relação à massa e distância entre objetos, sendo que a adaptação ao comércio se deu a partir da perspectiva de que o fluxo de comércio é diretamente influenciado pela renda dos países. Desta forma, o modelo gravitacional permite a definição de um esboço da área de interação de um país, próximo à ideia de área de mercado, levando em conta o poder de atração determinado positivamente pela intensidade das trocas e negativamente pela distância.

$$
T i j=(Y i \cdot Y j) / D i j
$$

onde,

Tij: Total do comércio entre pais i e pais $\mathrm{j}$

Yi e Yj: Renda do país (PIB)

Dij: Distancia entre i e j

De forma linearizada:

$$
\ln T_{i j}=\beta_{1}+\beta_{2} \ln Y_{i}+\beta_{3} \ln Y_{j}-\beta_{4} \ln D_{i j}
$$

Assim se espera que o comércio seja positivamente influenciado pelos PIBs e negativamente pela distância.

A justificativa teórica mais difundida para a utilização do modelo gravitacional remonta ao modelo de comércio desenvolvido por Krugman (1980), que considera que os consumidores buscam variedade ao consumir. Neste caso haveria diferenciação do produto entre as firmas monopolisticamente competitivas, não somente entre os países, e assim um país com maior produção teria maior capacidade de satisfazer os anseios dos consumidores ao ofertar uma ampla gama de produtos. Soma-se a isso o fato de as economias com grande produção tenderem a gastar muito com importações haja vista 
sua alta renda, desta forma o comércio entre duas economias seria maior, tanto maior fossem seus PIBs. Para justificar a influência da distância no modelo, os principais aspectos destacados são que a distância aumenta os custos de transporte, e, além disso, a comunicação e o fluxo de informações devido a diferenças culturais e idiomáticas aumentam os custos de informação acerca de leis, sistema tributário e outras regulamentações do país de destino, o que levaria países mais distantes a comerciar menos.

Este modelo, entretanto, apesar de apresentar um sucesso empírico em explicar o comércio bilateral, sofreu críticas por ser um modelo puramente matemático e intuitivo sem levar em conta fundamentos teóricos. Para dar mais credibilidade ao modelo, diversos pesquisadores tentaram fundamentá -lo, submetendo-o a extensões e alterações ao longo das décadas. Alterações estas aplicadas sob a intenção de adequar o modelo a outras análises.

Um exemplo disso é a critica de Anderson (1979) a respeito da simplicidade de análise e participação de variáveis, que faz tentar explicar teoricamente a equação gravitacional aplicada às commodities. Ele parte de um sistema onde cada país é especializado na produção de um único bem comercializável, e logo em seguida acrescenta um outro bem não comercializável no modelo. Por fim descarta essas hipóteses e aponta que vários produtos passam a ser comercializados se diferenciando apenas por locais de origem e a distância, negativamente inclinada ao fluxo de comércio, passa a determinar a quota de exportação entre eles.

Outra contribuição importante foi a de Bergstrand, 1985. Seu objetivo foi desenvolver uma fundamentação microeconômica para a equação gravitacional através das funções de utilidade e produção que gerariam a forma multiplicativa da equação. Para tanto utiliza duas suposições: que o mercado agregado entre dois países é relativamente pequeno quando comparado a outros mercados, e que os países tem funções de utilidade e produção idênticas, o que assegura o uso dos mesmos parâmetros para todos os países. Bergstrand tem o mérito de atribuir às variáveis e aos coeficientes interpretações econômicas concretas.

Como já mencionado, o modelo gravitacional é muito utilizado em estudos sobre o tema do comércio adaptado a objetivos diferentes. Exemplos disso são a tentativa de explicar o efeito-fronteira e a relevância da formação de blocos econômicos para o comércio. Com o intuito de 
aprimorá-lo, diversas outras variáveis já foram utilizadas, algumas explanatórias, como população (ou PIB per capita) e área do país, e algumas dummies $^{3}$ que representam características geográficas ou de proximidade cultural, como língua oficial, fronteira, cultura comum, participação de blocos comercias, entre outras.

\section{O Efeito-fronteira}

A globalização e a crescente integração entre os países desde o século passado definiram diversas mudanças na economia internacional. As barreiras que impediam um maior comércio entre os países do mundo foram, uma a uma, sendo ultrapassadas, e a integração e interdependência entre eles se mostrou cada vez mais evidente. A partir dos anos 90 iniciou-se um movimento com a finalidade de mensurar os impactos destas barreiras fronteiriças sobre o comércio tanto internacional como intranacional, denominado efeito-fronteira. Desde então surgiram estudos sobre o efeitofronteira, que foram feitos inicialmente para as economias norte-americanas e se estenderam para o Brasil e suas unidades federativas.

O propósito do cálculo consiste em mensurar o viés doméstico dos fluxos de comércio, ou seja, medir qual é o tamanho do comércio intranacional em contraposição ao comércio internacional, que consiste no efeito-fronteira. Para tanto, o modelo é constituído basicamente da Teoria Gravitacional de Newton adaptada para o comércio. Desta forma, tem-se que o comércio entre dois países é diretamente proporcional aos valores de seus produtos e inversamente à distância entre eles. $\mathrm{O}$ efeito-fronteira adiciona a este modelo variáveis binárias (dummies), que qualificam as observações e valem 1 para estados da federação e 0 para outros países. Assim, pode-se calcular utilizando os coeficientes estimados o viés de comércio entre os estados do país comparativamente com o externo.

Um dos estudos pioneiros no campo foi a regressão estimada por McCallum (1995), que por meio do Método de Mínimos Quadrados Ordinários, gerou resultados que evidenciaram tendência interna nos fluxos de comércio canadense:

Variáveis de caráter binário que indicam a presença ou ausência de uma "qualidade" ou atributo através dos valores I e 0, respectivamente (Gujarati, 2006). 


$$
\operatorname{Ln} X_{i j}=\beta_{0}+\beta_{1} \operatorname{Ln} Y_{i}+\beta_{2} \operatorname{Ln} Y_{j}+\operatorname{Ln}_{3} D I S T_{i j}+\beta_{4} D u m m y_{i j}+\varepsilon_{t}
$$

onde,

$\mathrm{X}_{\mathrm{ij}}$ : exportações nominais da província/estado i para a província/estado j; $\mathrm{Y}_{\mathrm{i}}$ e $\mathrm{Y}_{\mathrm{j}}$ : PIB nominal das província/estado i e j;

$\operatorname{DIST}_{\mathrm{ij}}$ : distância entre as província/estado i e $\mathrm{j}$;

Dummy ij $_{\text {: }}$ assume o valor de 1 no caso de exportações intraprovinciais do Canadá e zero para exportações de províncias canadenses para estados norte-americanos;

$\varepsilon_{\mathrm{ij}}$ : erro.

Ele selecionou as 10 províncias canadenses e outros 30 estados norte -americanos, e, através da estimação, observou que o comércio entre duas províncias canadenses é 22 vezes superior ao comércio entre províncias canadenses e estados americanos, de tamanho e distâncias similares, constatando a influência que as barreiras fronteiriças exerciam na época.

Mais tarde, Helliwell (1998) aplicou o mesmo modelo, visando analisar o comércio de Quebec (província canadense) com outras províncias do país e estados americanos. Constatou que entre Quebec e as províncias canadenses o comércio era 26 vezes superior que entre Quebec e os estados americanos de semelhante tamanho e distância.

Anderson e van Wincoop (2003) também utilizaram o exemplo da relação comercial entre Canadá e Estados Unidos, entretanto, propuseram uma equação gravitacional mais elaborada, alegando que as equações anteriores omitiam variáveis de grande importância para o modelo e adicionando-as com a finalidade de captar o custo de oportunidade, através de valores estimados de índices de resistência, que estariam diretamente conectados com as barreiras bilaterais. Também alegam que existe uma assimetria do efeito-fronteira entre países com tamanhos diferentes, com países menores apresentando efeitos maiores. Seu resíduo foi menor e a conclusão foi de que o comércio era, ao invés de 22 e 26,16 vezes maior.

Os autores atribuem a estes resultados os motivos de que o padrão de comércio do Canadá com os EUAé, em grande parte, explicado pelo tamanho reduzido da economia canadense em comparação à economia dos Estados Unidos; e também que o significativo valor encontrado do viés doméstico 
de comércio no Canadá se deve à ausência de variáveis que expressem a resistência multilateral ao comércio nos modelos anteriores.

Também foram realizados estudos na União Européia, dos quais se esperava que o viés de comércio doméstico fosse bastante reduzido ou insignificante, dado ao grande nível de integração dos países pertencentes ao bloco comercial. O que Nitsch (2000) constatou foi que no período entre 1979 e 1990 o comércio regional intra-bloco fora 10 vezes maior que o do bloco com outros países, reforçando a importância das fronteiras regionais para o comércio entre países pertencentes a blocos com elevado nível de integração.

Em 2007 houve a extensão destes estudos para o Brasil. Elaborado por Silva, Almeida e Oliveira, o estudo avaliava o viés comercial entre os estados brasileiros e entre estes e outros 40 países. Usando variáveis do ano de 1999, como o PIB per capita e a diferença entre PIBs per capita entre os estados e os países, os autores concluíram que o comércio no interior do país é 37,7 vezes maior do que o internacional, que foi justificado pelo baixo grau de substituição dos produtos brasileiros, além da existência de barreiras importantes que impediam o comércio entre o Brasil e outros países.

Constantini (2009) realizou um dos estudos que mais se assemelha com a proposta deste trabalho. $\mathrm{O}$ autor pretendeu mostrar o viés comercial que se constituía nas relações comerciais entre Paraná e os outros estados brasileiros, também utilizando dados do ano de 1999. Através do MQO e utilizando o PIB per capita, ele atingiu a proporção de 9,8 vezes que o Paraná comercializa com os outros estados brasileiros a mais do que comercializa com outros países.

\section{METODOLOGIA}

Com a finalidade de estimar os parâmetros do Modelo Gravitacional para a realidade brasileira utilizamos o Método dos Mínimos Quadrados Ordinários desenvolvido por Carl Friedrich Gauss segundo o qual a reta que melhor se ajusta aos dados, será aquela que minimiza a soma dos quadrados das distâncias verticais entre cada ponto e a Função de Regressão Amostral. É importante utilizar a distância ao quadrado para evitar que as grandes distâncias positivas e negativas sejam canceladas entre si, atribuindo devido peso aos que estão mais distantes da reta (HILL et al, 2003). Os estimadores de MQO, amplamente utilizados em regressões econômicas, financeiras e 
das ciências sociais em geral, apresentam propriedades teóricas aspiráveis. Tais propriedades estão contidas no teorema de Gauss-Markov, que afirma que o estimador de MQO é o melhor estimador do valor real do parâmetro se atende a quatro condições: é uma função linear de uma variável aleatória; não é tendencioso, seu valor esperado é igual ao verdadeiro; e possui variância mínima dentre os estimadores lineares não tendenciosos desse tipo.

O modelo utilizado no presente trabalho tem como variáveis independentes o PIB dos países/estados, a distância entre eles e o estado catarinense, e ainda uma variável dummy que assume valor 1 quando as mercadorias são exportadas de SC para outros Estados da Federação e valor 0 quando são exportadas para outros países.

$$
\ln E_{i j}=\beta_{1}+\beta_{2} \ln Y_{j}+\beta_{3} \ln D I S T_{i j}+\beta_{4} D
$$

onde, nesse caso:

$E_{\mathrm{ij}}$ : Exportação de Santa Catarina para estado ou país j

$\beta_{1}$ : Constante

$\mathrm{Y}_{\mathrm{j}}$ : PIB do Estado ou País j

DIST $_{\mathrm{ij}}$ : Distância entre Santa Catarina e o estado ou entre o Brasil (Brasília) e o país $\mathrm{j}$

D: variável dummy que assume o valor 1 se as exportações do Estado de Santa Catarina vão para o estado j do Brasil e 0 se elas têm como destino outro país;

j: País ou Estado com o qual Santa Catarina mantém comércio.

Espera-se que todos os estimadores, exceto $\beta_{3}$, assumam valores positivos.

No modelo em questão as variáveis dependentes são tanto as exportações entre o Estado de Santa Catarina e os demais 25 estados brasileiros e o Distrito Federal como para os países da amostra do ano de 2010. Os dados das exportações foram utilizados no lugar dos de fluxo de comércio pela indisponibilidade destes. Para os países em questão foram obtidos os dados referentes ao comércio da base de dados Aliceweb, do Ministério de Desenvolvimento, Indústria e Comércio Exterior (MDIC) a preços correntes de 2010 em dólares. Já entre Santa Catarina e os demais estados, os dados baseados na cobrança de ICMS foram obtidos da Secretaria da Fazenda de 
Santa Catarina. Os fluxos de comércio interestaduais dificilmente são encontrados, haja vista que o governo não possui o devido controle das mercadorias.

Os PIBs dos estados para 2010 ainda não tinham sido disponibilizados, desta forma, foi utilizada uma aproximação (proxy) calculada a partir do PIB total brasileiro em 2010, respeitando a participação de cada estado em 2009. Tanto o PIB dos estados como as exportações para estes foram encontrados em reais (R\$) correntes. Como os demais dados encontravam-se em dólares, os dados foram convertidos em dólares pela taxa média de câmbio em 2010 $(1,7594)$. Os PIBs dos países selecionados foram obtidos do Banco Mundial a preços correntes de 2010 em dólares. As distâncias entre Florianópolis e as capitais dos demais estados foram coletadas no Departamento Nacional de Infra-Estrutura de Transportes. Para os países, a distância utilizada é a compreendida entre Brasília e as demais capitais. Tais dados foram obtidos do Centre d'Etudes Prospectives et d'Informations Internationales (CEPII).

Foram encontrados 187 países com quem Santa Catarina comercializou em 2010. Contudo, alguns países ainda não possuíam o PIB correspondente de 2010 disponibilizado ou a distância calculada pelo CEPII. Além disso, existem países com comércio inexpressivo, por isso, utilizou-se um critério de comércio mínimo, ou seja, somente os países que apresentavam transações com Santa Catarina superiores a US\$ 100.000,00 foram incluídos na amostra. Com essas restrições, o número de observações passou a ser de 120 entre Santa Catarina e os países e 26 de comércio doméstico (25 estados mais o Distrito Federal).

A partir dos resultados da regressão, o efeito-fronteira pode ser calculado pela fórmula (Wooldridge, 2010):

onde:

$$
\mathrm{EF}=\frac{\mathrm{e}^{\mathrm{B} 1+\mathrm{B} 4}-\mathrm{e}^{\mathrm{B} 1}}{\mathrm{e}^{\mathrm{B} 1}}
$$

$\mathrm{B}_{1}=$ intercepto

$\mathrm{B}_{4}=$ coeficiente da variável Dummy.

\section{RESULTADOS}

Os resultados foram obtidos através do software gretl com uma amostra de 126 dados (países e estados). As tabelas com os valores utilizados se 
encontram no ANEXO deste artigo, onde A.1 contém os dados da distância de Santa Catarina aos demais estados, o valor de seus respectivos PIB, exportação e a dummy. A.2 apresenta esses mesmo dados para os 120 países incluídos na regressão.

Os valores da regressão se encontram na Tabela 1 abaixo, junto com os respectivos testes de significância corrigidos pelo método de White de correção de heterocedasticidade, problema bastante comum quando se trabalha com dados em corte transversal, mas que não altera o valor dos coeficientes.

Tabela 1 - Dados da regressão por MQO.

\begin{tabular}{lccccc} 
& & \multicolumn{2}{c}{$\begin{array}{c}\text { Antes da correção de } \\
\text { heterocedasticidade }\end{array}$} & \multicolumn{2}{c}{$\begin{array}{c}\text { Com correção de } \\
\text { eterocedasticidade }\end{array}$} \\
& Coeficiente & Teste - $\mathrm{t}$ & $\mathrm{P}-$ valor & Teste - $\mathrm{t}$ & P-Valor \\
\hline Cte & 5,39932 & 2,742 & 0,0069 & 2,891 & 0,0045 \\
$\mathrm{LnY}_{\mathrm{j}}$ & 0,814366 & 16,16 & $5,59 \mathrm{e}-34$ & 18,09 & $1,14 \mathrm{e}-38$ \\
$\mathrm{LnD}_{\mathrm{ij}}$ & $-1,06445$ & $-5,536$ & $1,45 \mathrm{e}-07$ & $-5,870$ & $2,94 \mathrm{e}-08$ \\
$\mathrm{D}$ & 3,49467 & 2,742 & $4,51 \mathrm{e}-17$ & 11,00 & $9,68 \mathrm{e}-21$ \\
\hline
\end{tabular}

Fonte: gretl com base nos dados selecionados.

Com base na regressão obtida, observa-se que o modelo comportou-se de acordo com o esperado, uma vez que todos os coeficientes, excetuando a distância, foram positivos. $O$ teste de significância individual (teste $t$ ) foi significativo para todos os parâmetros a menos de $1 \%$, o que garante sua importância no modelo.

Tabela 2 - Teste F e P-Valor

\begin{tabular}{ccc} 
& $\begin{array}{c}\text { Antes da correção de } \\
\text { heterocedasticidade }\end{array}$ & $\begin{array}{c}\text { Com correção de } \\
\text { heterocedasticidade }\end{array}$ \\
\hline Teste-F $(3,142)$ & 186,4546 & 203,157 \\
P - valor (F) & $4,81 \mathrm{e}-49$ & $3,62 \mathrm{e}-51$ \\
\hline
\end{tabular}

Fonte: gretl com base nos dados selecionados. 
Na Tabela 2 observa-se que, com base no teste de significância global (teste F), os parâmetros em conjunto também são significativos a menos de $1 \%$. Apesar do modelo ter apresentado heterocedasticidade, as correções realizadas observadas nas Tabelas 1 e 2, não alteram a significância tanto individual quanto do modelo como um todo. Também não foi constatada a presença de multicolinearidade no modelo. $\mathrm{O}^{2}$ ajustado no valor de 0,79326 representa que $79 \%$ das exportações podem ser explicadas pelo modelo.

Com estas informações, é possível calcular o efeito-fronteira referente às exportações de Santa Catarina no ano de 2010. O efeito-fronteira apresentou um valor estimado aproximado de 32, ou seja, o comércio entre Santa Catarina e um estado brasileiro é aproximadamente 32 vezes maior em comparação a um país com o mesmo PIB e mesma distância.

\section{CONCLUSÃO}

Como principal conclusão deste trabalho pode-se destacar a maior importância para o estado de Santa Catarina do comércio com outros entes federativos do Brasil, do que com os demais países. Assim, pelo fato do parceiro comercial se encontrar dentro da mesma fronteira internacional, Santa Catarina comercializa cerca de 32 vezes mais com este, que com o mesmo, caso se encontrasse fora.

O resultado obtido já era esperado na medida em que o efeito-fronteira apresenta valores históricos positivos, contudo sua magnitude foi maior do que o esperado. O estudo realizado por Silva, Almeida e Oliveira, 2007, sobre a conjuntura de 1999, mostrou que o efeito-fronteira para todo o mercado brasileiro era maior do que o encontrado para Santa Catarina em 2010, o fluxo de comercio doméstico se mostrava 37,7 vezes maior que o externo. Já o estudo do Paraná mostrou um efeito muito menor, de 9,8. Esperava-se que o resultado para Santa Catarina fosse mais próximo do paranaense, devido a semelhanças no comportamento do mercado e já que a região sul em geral possui um grau maior de internacionalização do que o resto do Brasil.

O que pode explicar parte desse valor relativamente elevado é o fato de Santa Catarina ainda guardar uma peculiaridade em relação a cadeia produtiva em que está inserida. Apesar de possuir uma indústria de metalmecânica e de têxteis bastante competitivas, grande parte desses produtos 
não são vendidos diretamente ao consumidor final, mas sim repassados a outras indústrias, normalmente em outros estado, para sua finalização. Assim, outros setores importantes da economia catarinense, ligados a agroindústria, mineração, bens de pouco valor agregado e exportado pelo estado em grande quantidade.

Outro ponto relevante diz respeito a conjuntura internacional. Em 2010 o mercado internacional passou por uma fase difícil devido a crise do fim de 2008, isso provavelmente influenciou o viés do comercio catarinense para cima. Juntamente com a queda do mercado externo, teve-se um fortalecimento do interno, com o crescimento da classe média e incentivos governamentais para o aumento do consumo, o que levou os demais países a comprarem menos enquanto e o mercado interno a comprar mais.

\title{
BORDER EFFECT: AN ANALYSIS OF TRADE IN SANTA CATARINA
}

\begin{abstract}
This article aims to analyze the border effect for the state of Santa Catarina compare to 25 states and the Federal District of Brazil and to other countries in 2010. Therefore, was used data on exports of Santa Catarina, the GDP of other states and countries and the distance between they and the state of Santa Catarina to the development of an estimation model that uses dummy variables to adjust the model of gravitational trade to the analysis of the effect border. From the estimated regression was founded that trade between Santa Catarina and the Brazilian state is about 32 times higher when compared to a country with the same GDP and same distance.
\end{abstract}

Keywords: border effect, model of gravitational trade, international trade, trade between states, Santa Catarina.

JEL Classification: C2: F1. 


\section{REFERÊNCIAS}

ANDERSON, James E.; WINCOOP, Eric van. Gravity with Gravitas: A Solution to the Border Puzzle. American Economic Review, AER, vol. 93(1), pages 170-192; 2003.

CONSTANTINI, Jaime Jordan. Ensaios sobre instituições, crescimento e distribuição. Início: 2009. Tese (Doutorado em Desenvolvimento Econômico) - Universidade Federal do Paraná, Coordenação de Aperfeiçoamento de Pessoal de Nível Superior. Disponível em: http://www.ecopar.ufpr.br/ artigos/a7_036.pdf. Acesso em: 16/02/12.

GUJARATI, D. N. Econometria básica. Rio de Janeiro: Elsevier, 2006. $812 \mathrm{p}$

HELLIWELL, John F. How Much Do National Borders Matter? Washington DC: Brookings Institution Press, v. 156; 1998.

HILL, R. Carter; GRIFFITHS, William E.; JUDGE, George G . Econometria. 2. ed São Paulo (SP): Saraiva, 2003. 471p.

McCALLUM, John. National Borders Matter: Canada-U.S. Regional Trade Patterns; The American Economic Review, Vol. 85, No. 3, pp. 615-623; 1995.

NITSCH, Volker. National Borders and International Trade: Evidence from the European Union. Canadian Journal of Economics. 33 (November): 1091-1105; 2000.

POYHONEN, P. A Tentative Model for the Volume of Trade Between Countries. Weltwirtschaftliches Archive 90, 93-100; 1963.

SILVA, O. M; ALMEIDA, F. M.; OLIVEIRA, B. M. Intra-national versus international trade in Brazil: measuring the border effect. XII Annual Conference. Western Hemispheric Integration in a Competitive Global Environment, 2007, Texas and Monterrey, Anais, Loredo: TAMIU; 2007. 
TINBERGEN, Jan. Shaping the world economy Suggestions for an international economic policy. New York: Twentieth Century Fund; 1962.

WOOLDRIDGE, Jeffrey M. Introdução à Econometria: uma abordagem moderna. São Paulo: Cengage, 2010. 701 p.

\section{RESULTADOS}

Tabela A1 - Distância de Santa Catarina aos demais Estados, PIB, exportação e dummy.

\begin{tabular}{|c|c|c|c|c|}
\hline Estado & dist & PIB & ex & dummy \\
\hline $\mathrm{AC}$ & 3976 & $4,76 \mathrm{E}+09$ & 72220510 & 1 \\
\hline $\mathrm{AL}$ & 3168 & $1,37 \mathrm{E}+10$ & $2,66 \mathrm{E}+08$ & 1 \\
\hline AM & 4443 & $3,2 \mathrm{E}+10$ & $3,98 \mathrm{E}+08$ & 1 \\
\hline AP & 3083 & $4,77 \mathrm{E}+09$ & 92149673 & 1 \\
\hline $\mathrm{BA}$ & 2682 & $8,84 \mathrm{E}+10$ & $1,63 \mathrm{E}+09$ & 1 \\
\hline $\mathrm{CE}$ & 3838 & $4,24 \mathrm{E}+10$ & $9,39 \mathrm{E}+08$ & 1 \\
\hline DF & 1673 & $8,48 \mathrm{E}+10$ & $7,48 \mathrm{E}+08$ & 1 \\
\hline ES & 1597 & $4,3 \mathrm{E}+10$ & $1,14 \mathrm{E}+09$ & 1 \\
\hline $\mathrm{GO}$ & 1493 & $5,52 \mathrm{E}+10$ & $1,72 \mathrm{E}+09$ & 1 \\
\hline MA & 3537 & $2,57 \mathrm{E}+10$ & $3,82 \mathrm{E}+08$ & 1 \\
\hline $\mathrm{MG}$ & 1301 & $1,85 \mathrm{E}+11$ & $4,07 \mathrm{E}+09$ & 1 \\
\hline MS & 1298 & $2,35 \mathrm{E}+10$ & $7,75 \mathrm{E}+08$ & 1 \\
\hline MT & 1986 & $3,69 \mathrm{E}+10$ & $1 \mathrm{E}+09$ & 1 \\
\hline PA & 3500 & $3,77 \mathrm{E}+10$ & $6,8 \mathrm{E}+08$ & 1 \\
\hline PB & 3485 & $1,85 \mathrm{E}+10$ & $3,62 \mathrm{E}+08$ & 1 \\
\hline $\mathrm{PE}$ & 3375 & $5,06 \mathrm{E}+10$ & $1,44 \mathrm{E}+09$ & 1 \\
\hline PI & 3450 & $1,23 \mathrm{E}+10$ & $2,4 \mathrm{E}+08$ & 1 \\
\hline PR & 300 & $1,23 \mathrm{E}+11$ & $1,5 \mathrm{E}+10$ & 1 \\
\hline $\mathrm{RJ}$ & 1144 & $2,28 \mathrm{E}+11$ & $2,96 \mathrm{E}+08$ & 1 \\
\hline $\mathrm{RN}$ & 3662 & $1,8 \mathrm{E}+10$ & $1 \mathrm{E}+10$ & 1 \\
\hline RO & 3442 & $1,3 \mathrm{E}+10$ & $4,38 \mathrm{E}+08$ & 1 \\
\hline RR & 5128 & $3,61 \mathrm{E}+09$ & 38461001 & 1 \\
\hline $\mathrm{RS}$ & 476 & $1,39 \mathrm{E}+11$ & $4,66 \mathrm{E}+09$ & 1 \\
\hline SE & 2892 & $1,27 \mathrm{E}+10$ & $2,32 \mathrm{E}+08$ & 1 \\
\hline SP & 705 & $6,99 E+11$ & $2,93 \mathrm{E}+10$ & 1 \\
\hline TO & 2336 & $9,4 \mathrm{E}+09$ & $1,79 \mathrm{E}+08$ & 1 \\
\hline
\end{tabular}


Tabela A2 - Distância de Santa Catarina aos países, PIB, exportação e dummy.

\begin{tabular}{|c|c|c|c|c|}
\hline País & dist & PIB & ex & dummy \\
\hline Albania & 9943,985 & $1,18 \mathrm{E}+10$ & 4196011 & 0 \\
\hline Algeria & 8497,729 & $1,59 \mathrm{E}+11$ & 3992322 & 0 \\
\hline Angola & 6559,966 & $8,44 \mathrm{E}+10$ & 71585218 & 0 \\
\hline Antigua and Barbuda & 4818,193 & $1,01 \mathrm{E}+09$ & 2194356 & 0 \\
\hline Argentina & 1691,067 & $3,69 \mathrm{E}+11$ & $5,5 \mathrm{E}+08$ & 0 \\
\hline Armenia & 11769,69 & $9,26 \mathrm{E}+09$ & 5588025 & 0 \\
\hline Austria & 10146,47 & $3,76 \mathrm{E}+11$ & 8500552 & 0 \\
\hline Azerbaijan & 12214,38 & $5,11 \mathrm{E}+10$ & 6504244 & 0 \\
\hline Bahamas & 6343,316 & $7,54 \mathrm{E}+09$ & 4344443 & 0 \\
\hline Bangladesh & 15665,7 & $1 \mathrm{E}+11$ & 8026802 & 0 \\
\hline Barbados & 4315,083 & $3,2 \mathrm{E}+09$ & 2295931 & 0 \\
\hline Belarus & 11135,23 & $5,47 \mathrm{E}+10$ & 4569442 & 0 \\
\hline Belize & 6447,859 & $1,43 \mathrm{E}+09$ & 453005 & 0 \\
\hline Benin & 6291,807 & $6,63 \mathrm{E}+09$ & 3967275 & 0 \\
\hline Bolivia & 2380,92 & $1,98 \mathrm{E}+10$ & 58233829 & 0 \\
\hline Bosnia and Herzegovina & 10003,96 & $1,69 \mathrm{E}+10$ & 1915940 & 0 \\
\hline Bulgaria & 10267,08 & $4,77 \mathrm{E}+10$ & 8875330 & 0 \\
\hline Burkina Faso & 6314,805 & $8,82 \mathrm{E}+09$ & 205769 & 0 \\
\hline Cameroon & 6997,694 & $2,24 \mathrm{E}+10$ & 320909 & 0 \\
\hline Canada & 8191,353 & $1,57 \mathrm{E}+12$ & 62104835 & 0 \\
\hline Cape Verde & 4964,447 & $1,65 \mathrm{E}+09$ & 1470194 & 0 \\
\hline Chad & 7791,363 & $7,59 \mathrm{E}+09$ & 109207 & 0 \\
\hline Chile & 2590,783 & $2,03 \mathrm{E}+11$ & $1,43 \mathrm{E}+08$ & 0 \\
\hline China & 17614,3 & $5,88 \mathrm{E}+12$ & $2,71 \mathrm{E}+08$ & 0 \\
\hline Colombia & 4399,134 & $2,88 \mathrm{E}+11$ & 58114931 & 0 \\
\hline Comoros & 9491,185 & $5,41 \mathrm{E}+08$ & 136553 & 0 \\
\hline Costa Rica & 5523,376 & $3,46 \mathrm{E}+10$ & 10263059 & 0 \\
\hline Croatia & 9966,041 & $6,09 \mathrm{E}+10$ & 10567413 & 0 \\
\hline Czech Republic & 10155,45 & $1,92 \mathrm{E}+11$ & 1903882 & 0 \\
\hline Denmark & 10431,67 & $3,1 \mathrm{E}+11$ & 11399543 & 0 \\
\hline Dominican Republic & 5311,118 & $5,16 \mathrm{E}+10$ & 11789522 & 0 \\
\hline Ecuador & 4309,081 & $5,89 \mathrm{E}+10$ & 43046999 & 0 \\
\hline Egypt & 10229,25 & $2,19 \mathrm{E}+11$ & 74325446 & 0 \\
\hline El Salvador & 6210,195 & $2,18 \mathrm{E}+10$ & 3688180 & 0 \\
\hline
\end{tabular}




\begin{tabular}{c|c|c|c|c}
\hline País & dist & PIB & ex & dummy \\
\hline Equatorial Guinea & 6729,316 & $1,4 \mathrm{E}+10$ & 3724980 & 0 \\
\hline Estonia & 11266,82 & $1,87 \mathrm{E}+10$ & 686688 & 0 \\
\hline Ethiopia & 9960,68 & $2,97 \mathrm{E}+10$ & 2605124 & 0 \\
\hline Finland & 11316,05 & $2,39 \mathrm{E}+11$ & 6361108 & 0 \\
\hline France & 9408,406 & $2,56 \mathrm{E}+12$ & $1,43 \mathrm{E}+08$ & 0 \\
\hline Gabon & 6608,553 & $1,3 \mathrm{E}+10$ & 2638117 & 0 \\
\hline Georgia & 11846,2 & $1,17 \mathrm{E}+10$ & 6717271 & 0 \\
\hline Germany & 9847,663 & $3,31 \mathrm{E}+12$ & $3,05 \mathrm{E}+08$ & 0 \\
\hline Ghana & 5985,302 & $3,13 \mathrm{E}+10$ & 12043382 & 0 \\
\hline Greece & 10036,02 & $3,05 \mathrm{E}+11$ & 9636961 & 0 \\
\hline Grenada & 4289,566 & $6,28 \mathrm{E}+08$ & 2613662 & 0 \\
\hline Guatemala & 6390,672 & $4,12 \mathrm{E}+10$ & 15712824 & 0 \\
\hline Guinea & 4984,344 & $4,51 \mathrm{E}+09$ & 147426 & 0 \\
\hline Guyana & 3597,721 & $2,22 \mathrm{E}+09$ & 2557611 & 0 \\
\hline Haiti & 5452,449 & $6,71 \mathrm{E}+09$ & 5298816 & 0 \\
\hline Honduras & 6086,675 & $1,54 \mathrm{E}+10$ & 7191605 & 0 \\
\hline Hong Kong & 18059,72 & $2,24 \mathrm{E}+11$ & $2,04 \mathrm{E}+08$ & 0 \\
\hline Hungary & 10267,27 & $1,3 \mathrm{E}+11$ & 20017057 & 0 \\
\hline Iceland & 9991,34 & $1,26 \mathrm{E}+10$ & 111014 & 0 \\
\hline India & 14441,49 & $1,73 \mathrm{E}+12$ & 34393320 & 0 \\
\hline Indonesia & 15644,48 & $7,07 \mathrm{E}+11$ & 21668283 & 0 \\
\hline Iraq & 11520,45 & $8,22 \mathrm{E}+10$ & 36686489 & 0 \\
\hline Ireland & 9398,416 & $2,04 \mathrm{E}+11$ & 19002019 & 0 \\
\hline Israel & 10629,06 & $2,17 \mathrm{E}+11$ & 7692308 & 0 \\
\hline Italy & 9482,516 & $2,05 \mathrm{E}+12$ & $1,59 \mathrm{E}+08$ & 0 \\
\hline Jamaica & 5663,679 & $1,4 \mathrm{E}+10$ & 8248604 & 0 \\
\hline Japan & 18549,61 & $5,5 \mathrm{E}+12$ & $4,79 \mathrm{E}+08$ & 0 \\
\hline Jordan & 10723,49 & $2,76 \mathrm{E}+10$ & 10725976 & 0 \\
\hline Kenya & 9292,935 & $3,14 \mathrm{E}+10$ & 2762682 & 0 \\
\hline Kyrgyzstan & 14281,39 & $4,62 \mathrm{E}+09$ & 1420414 & 0 \\
\hline Latvia & 11103,81 & $2,4 \mathrm{E}+10$ & 1916218 & 0 \\
\hline Lebanon & 10772,67 & $3,92 \mathrm{E}+10$ & 4842201 & 0 \\
\hline Liberia & 5121,55 & $9,86 \mathrm{E}+08$ & 992800 & 0 \\
\hline Lithuania & 11051,35 & $3,63 \mathrm{E}+10$ & 5440941 & 0 \\
\hline Luxembourg & 9663,413 & $5,51 \mathrm{E}+10$ & 470149 & 0 \\
\hline Madagascar & 9591,817 & $8,72 \mathrm{E}+09$ & 607181 & 0 \\
\hline Malaysia & 15940,63 & $2,38 \mathrm{E}+11$ & 17424908 & 0 \\
\hline Maldives & 13264,65 & $1,48 \mathrm{E}+09$ & 1907714 & 0 \\
\hline Mauritania & 5711,193 & $3,64 \mathrm{E}+09$ & 1516037 & 0 \\
\hline & & & & \\
\hline & & & \\
\hline & & & \\
\hline & &
\end{tabular}




\begin{tabular}{|c|c|c|c|c|}
\hline País & dist & PIB & ex & dummy \\
\hline Mauritius & 10479,45 & $9,73 \mathrm{E}+09$ & 1400321 & 0 \\
\hline Mexico & 7439,636 & $1,04 \mathrm{E}+12$ & $2,87 \mathrm{E}+08$ & 0 \\
\hline Morocco & 7665,229 & $9,12 \mathrm{E}+10$ & 18531642 & 0 \\
\hline Mozambique & 7878,605 & $9,59 \mathrm{E}+09$ & 3364471 & 0 \\
\hline Namibia & 6470,25 & $1,22 \mathrm{E}+10$ & 4542491 & 0 \\
\hline Netherlands & 9810,82 & $7,83 \mathrm{E}+11$ & $6,34 \mathrm{E}+08$ & 0 \\
\hline Nicaragua & 5862,436 & $6,55 \mathrm{E}+09$ & 2536240 & 0 \\
\hline Nigeria & 6382,416 & $1,94 \mathrm{E}+11$ & 18196842 & 0 \\
\hline Norway & 10641,01 & $4,14 \mathrm{E}+11$ & 1023339 & 0 \\
\hline Pakistan & 14121,48 & $1,75 \mathrm{E}+11$ & 4801169 & 0 \\
\hline Panama & 5084,887 & $2,68 \mathrm{E}+10$ & 12914629 & 0 \\
\hline Paraguay & 1134,649 & $1,85 \mathrm{E}+10$ & $1,67 \mathrm{E}+08$ & 0 \\
\hline Peru & 3455,375 & $1,54 \mathrm{E}+11$ & 57890086 & 0 \\
\hline Philippines & 18396,48 & $2 \mathrm{E}+11$ & 34276590 & 0 \\
\hline Poland & 10668,76 & $4,69 \mathrm{E}+11$ & 72096215 & 0 \\
\hline Portugal & 7955,642 & $2,29 \mathrm{E}+11$ & 46295753 & 0 \\
\hline Russian Federation & 11820,15 & $1,48 \mathrm{E}+12$ & $2,2 \mathrm{E}+08$ & 0 \\
\hline Saint Lucia & 4461,426 & $9,32 \mathrm{E}+08$ & 664272 & 0 \\
\hline Saudi Arabia & 11404,51 & $4,35 \mathrm{E}+11$ & $1,47 \mathrm{E}+08$ & 0 \\
\hline Senegal & 5303,289 & $1,3 \mathrm{E}+10$ & 2491677 & 0 \\
\hline Seychelles & 11036,87 & $9,37 \mathrm{E}+08$ & 1343743 & 0 \\
\hline Sierra Leone & 5085,813 & $1,91 \mathrm{E}+09$ & 465633 & 0 \\
\hline Singapore & 16000,61 & $2,23 \mathrm{E}+11$ & $1,31 \mathrm{E}+08$ & 0 \\
\hline Slovakia & 10191,96 & $8,9 \mathrm{E}+10$ & 9357544 & 0 \\
\hline Slovenia & 9891,706 & $4,78 \mathrm{E}+10$ & 254293 & 0 \\
\hline South Africa & 6355,119 & $3,64 \mathrm{E}+11$ & $1,88 \mathrm{E}+08$ & 0 \\
\hline Spain & 8389,78 & $1,41 \mathrm{E}+12$ & 97615466 & 0 \\
\hline Sri Lanka & 14036,67 & $4,96 \mathrm{E}+10$ & 1242360 & 0 \\
\hline Sudan & 9640,195 & $6,2 \mathrm{E}+10$ & 1389110 & 0 \\
\hline Swaziland & 7728,15 & $3,65 \mathrm{E}+09$ & 227686 & 0 \\
\hline Sweden & 10936,15 & $4,58 \mathrm{E}+11$ & 37303998 & 0 \\
\hline Switzerland & 9534,491 & $5,24 \mathrm{E}+11$ & 23495722 & 0 \\
\hline Tajikistan & 13787,02 & $5,64 \mathrm{E}+09$ & 867711 & 0 \\
\hline Thailand & 16409,97 & $3,19 \mathrm{E}+11$ & 10382398 & 0 \\
\hline Togo & 6170,232 & $3,15 \mathrm{E}+09$ & 1105093 & 0 \\
\hline Trinidad and Tobago & 4133,307 & $2,04 \mathrm{E}+10$ & 14372350 & 0 \\
\hline Tunisia & 8979,379 & $4,43 \mathrm{E}+10$ & 2240846 & 0 \\
\hline Turkey & 10590,76 & $7,35 \mathrm{E}+11$ & 65882943 & 0 \\
\hline Uganda & 8932,303 & $1,7 \mathrm{E}+10$ & 258425 & 0 \\
\hline
\end{tabular}




\begin{tabular}{c|c|c|c|c}
\hline País & dist & PIB & ex & dummy \\
\hline Ukraine & 11155,24 & $1,38 \mathrm{E}+11$ & 87704591 & 0 \\
\hline United Kingdom & 9501,71 & $2,25 \mathrm{E}+12$ & $3 \mathrm{E}+08$ & 0 \\
\hline United States of America & 7694,307 & $1,46 \mathrm{E}+13$ & $9,06 \mathrm{E}+08$ & 0 \\
\hline Uruguay & 1567,878 & $4,03 \mathrm{E}+10$ & $1,27 \mathrm{E}+08$ & 0 \\
\hline Uzbekistan & 13836,31 & $3,9 \mathrm{E}+10$ & 10293732 & 0 \\
\hline Venezuela & 4393,217 & $3,88 \mathrm{E}+11$ & $1,17 \mathrm{E}+08$ & 0 \\
\hline Zambia & 7826,946 & $1,62 \mathrm{E}+10$ & 267508 & 0 \\
\hline Zimbabwe & 8017,05 & $7,47 \mathrm{E}+09$ & 122491 & 0 \\
\hline
\end{tabular}

